\title{
Kinetics and correlates of the neutralizing antibody response to SARS-CoV-2
}

\section{Florian Klein ( $\nabla$ florian.klein@uk-koeln.de)}

Laboratory of Experimental Immunology, Institute of Virology, Faculty of Medicine and University Hospital of Cologne, University of Cologne https://orcid.org/0000-0003-1376-1792

\section{Kanika Vanshylla}

Laboratory of Experimental Immunology, Institute of Virology, Faculty of Medicine and University Hospital of Cologne, University of Cologne, Cologne

\section{Veronica Cristanziano}

Institute of Virology, Faculty of Medicine and University Hospital of Cologne,University of Cologne Franziska Kleipass

Institute of Virology, Faculty of Medicine and University Hospital of Cologne,University of Cologne

Felix Dewald

University Hospital Cologne

\section{Philipp Schommers}

Laboratory of Experimental Immunology, Institute of Virology, Faculty of Medicine and University Hospital of Cologne, University of Cologne

\section{Lutz Gieselmann}

Laboratory of Experimental Immunology, Institute of Virology, Faculty of Medicine and University Hospital of Cologne, University of Cologne

\section{Henning Gruell}

University of Cologne https://orcid.org/0000-0002-0725-7138

\section{Maike Schlotz}

Institute of Virology, Faculty of Medicine and University Hospital of Cologne,University of Cologne

\section{Meryem Ercanoglu}

Laboratory of Experimental Immunology, Institute of Virology, Faculty of Medicine and University Hospital of Cologne, University of Cologne

\section{Ricarda Stumpf}

Institute of Virology, Faculty of Medicine and University Hospital of Cologne,University of Cologne

\section{Petra Mayer}

University of Cologne

\section{Eva Heger}

Institute of Virology, Faculty of Medicine and University Hospital of Cologne,University of Cologne Wibke Johannis 
Institute for Clinical Chemistry, Faculty of Medicine and University Hospital Cologne, University of Cologne

\section{Carola Horn}

Department I of Internal Medicine, Medical Faculty and University Hospital Cologne, University of Cologne

\section{Isabelle Suárez}

University Hospital Cologne

\section{Norma Jung}

Department I of Internal Medicine, Medical Faculty and University Hospital Cologne, University of Cologne

\section{Susanne Salomon}

Institute of Virology, Faculty of Medicine and University Hospital of Cologne,University of Cologne https://orcid.org/0000-0002-5143-2351

\section{Kirsten Eberhardt}

Department of Tropical Medicine, Bernhard Nocht Institute for Tropical Medicine and Department of Medicine, University Medical Center Hamburg-Eppendorf https://orcid.org/0000-0002-1002-0326

\section{Gerd Fätkenheuer}

University Hospital Cologne

\section{Nico Pfeifer}

University of Tübingen

\section{Ralf Eggeling}

Methods in Medical Informatics, Department of Computer Science, University of Tübingen

\section{Max Augustin}

University Hospital Cologne, University of Cologne https://orcid.org/0000-0002-2300-9337

\section{Clara Lehmann}

University of Cologne

\section{Article}

Keywords: SARS-CoV2, COVID-19, antibodies, immunology

Posted Date: February 1st, 2021

DOl: https://doi.org/10.21203/rs.3.rs-148929/v1

License: (c) (1) This work is licensed under a Creative Commons Attribution 4.0 International License. Read Full License 


\section{Abstract}

A detailed understanding of antibody-based SARS-CoV-2 immunity has critical implications for overcoming the COVID-19 pandemic and for informing on vaccination strategies. In this study, we evaluated the dynamics of the SARS-CoV-2 antibody response in a cohort of 963 recovered individuals over a period of 10 months. Investigating a total of 2,146 samples, we detected an initial SARS-CoV-2 antibody response in $94.4 \%$ of individuals, with $82 \%$ and $79 \%$ exhibiting serum and IgG neutralization, respectively. Approximately $3 \%$ of recovered patients demonstrated exceptional SARS-CoV-2 neutralizing activity, defining them as 'elite neutralizers'. These individuals also possessed effective cross-neutralizing IgG antibodies to SARS-CoV-1 without any known prior exposure to this virus. By applying multivariate statistical modeling, we found that sero-reactivity, age, time since disease onset, and fever are key factors predicting SARS-CoV-2 neutralizing activity in mild courses of COVID-19. Investigating longevity of the antibody response, we detected loss of anti-spike reactivity in 13\% of individuals 10 months after infection. Moreover, neutralizing activity had an initial half-life of 6.7 weeks in serum versus 30.8 weeks in purified IgG samples indicating the presence of a more stable and long-term memory lgG B cell repertoire in the majority of individuals recovered from COVID-19. Our results demonstrate a broad spectrum of the initial SARS-CoV-2 neutralizing antibody response depending on clinical characteristics, with antibodies being maintained in the majority of individuals for the first 10 months after mild course of COVID-19.

\section{Main}

COVID-19 is caused by the severe acute respiratory syndrome coronavirus 2 (SARS-CoV-2), which was first identified in December 2019 1,2 . Since then, the virus has rapidly spread across the globe and caused more than 90 million proven infections and over 2 million deaths. Disease severity ranges from asymptomatic infection to symptoms like cough, fever, muscle pain, and diarrhea to severe courses of infection including pneumonia with severe respiratory distress and a high risk of death ${ }^{3-5}$. While the majority of infected individuals experience a mild course of disease, elderly or individuals with preexisting conditions are at higher risk for severe courses of COVID- $19^{6}$. In symptomatic non-hospitalized cases, the acute course of disease typically spans 7-14 days ${ }^{7,8}$. However, a significant fraction of COVID19 patients suffer long-lasting symptoms post recovery, so called 'post-COVID syndrome' ${ }^{\prime-11}$ (Augustin et al., submitted).

SARS-CoV-2 infects human cells by using the virus spike (S) protein ${ }^{12}$ for targeting the angiotensin converting enzyme-2 (ACE-2) receptor ${ }^{13}$. The S-protein carries dominant epitopes against which humoral $B$ and $T$ cell responses are generated upon natural infection and vaccination ${ }^{14-18}$. Spike-specific $\lg M, \lg A$, and IgG antibodies are detected early after infection ${ }^{19,20}$ and $\lg G$ antibody levels and $\lg G$ memory $B$ cells can persist post infection ${ }^{21}$.

Neutralizing antibodies (NAbs) are powerful molecules that target viruses and block infection. Moreover, they can eliminate circulating viruses and infected cells by antibody-mediated effector functions ${ }^{22,23}$. As 
a result, NAbs are crucial to overcome infectious diseases and are an important correlate of protection ${ }^{24}$. For SARS-CoV-2, vaccine induced NAbs as well as purified IgGs from convalescent animals have been shown to protect non-human primates (NHPs) from infection in a SARS-CoV-2 challenge model 25,26. Moreover, highly potent monoclonal NAbs have been isolated ${ }^{27-29}$ and are being used for treatment of COVID-19 in humans ${ }^{30,31}$.

Given the short time SARS-CoV-2 has been studied, information on long-term antibody dynamics are limited. Recent studies show that serum neutralizing activity is detectable within a week after onset of symptoms ${ }^{32,33}$ and can persist for the first months after infection ${ }^{21,23,34}$. Moreover, studies with symptomatic and hospitalized individuals have shown that more severe courses of disease result in a stronger SARS-CoV-2 neutralizing antibody response ${ }^{14,35,36}$. While these studies provide important insights, a precise quantification of SARS-CoV-2 neutralizing activity and dynamics as well as clinical correlates of developing a protective antibody response are largely unknown.

In this study, we set out to provide a deeper understanding of the neutralizing antibody response to SARSCoV-2. To this end, we determined neutralizing serum and IgG activity of 2,146 samples from a longitudinally monitored cohort of 963 individuals over time together with detailed information on the course of disease and past medical history. We combined statistical modeling to infer antibody decay rates after SARS-CoV-2 infection and built a prediction model for evaluating how clinical or disease features correlate with NAb titers. Finally, we performed longitudinal analyses to study anti-spike antibody levels as well as NAb titers for a time period of up to 10 months post SARS-CoV-2 infection. Our results inform on the kinetics, longevity and features affecting the antibody response to SARS-CoV-2. They are critical to understand SARS-CoV-2 immunity and to guide non-pharmacological interventions and vaccination strategies to overcome COVID-1937.

\section{Results}

\section{Establishing a cohort for investigating SARS-CoV-2 immunity}

To investigate the development of SARS-CoV-2 immunity, we established a cohort of COVID-19 patients who recently recovered from SARS-CoV-2 infection. Time since disease onset was derived from selfreported symptom onset or date of positive naso-/oro-pharyngeal swab. In addition, each participant reported details on the course of infection, symptoms, and past medical history (Supplementary Table 1). Participants enrolled ranged from 18-79 years of age (median: 44 years) with a balanced distribution of males (46.1\%) and females (53.9\%). Disease severity included asymptomatic (4.6\%), mildly symptomatic (91.69\%), and hospitalized individuals (2.6\%; Fig. 1, Supplementary Table 1). 23.4\% of participants reported pre-existing conditions that have been described to influence COVID-19 outcomes ${ }^{6}$.

Blood samples were collected from 963 individuals at study visit 1 (median 7.3 weeks post disease onset) with follow up analyses at study visit 2 for 616 participants (median 18.8 weeks post disease onset), study visit 3 for 430 participants (median 30.1 weeks post disease onset), and study visit 4 for 
137 participants (median 37.9 weeks post disease onset; Fig. 1). Other participants were lost in follow-up or did not reach the respective study visit at the time of our analysis. Anti-spike IgG was quantified by ELISA and chemiluminescent immunoassays (CLIA) and the NAb response to SARS-CoV-2 was analyzed using both serum dilutions as well as purified IgG to precisely quantify neutralizing activity (Extended Data Fig. 1). In total, 4,516 measurements were collected for visit 1 with another 1,867 subsequent measurements for visit 2-4 to determine the SARS-CoV-2 antibody response for 10 months following infection.

\section{Broad spectrum of the initial SARS-CoV-2 neutralizing antibody response}

NAb levels were quantified by testing serum and purified IgG from plasma/serum against pseudovirus particles expressing the Wuhan01 spike protein (EPI_ESL406716). Serum neutralization at study visit 1 was categorized based on titer into non- $\left(\mathrm{ID}_{50}<10\right)$, low- $\left(\mathrm{ID}_{50}=10-25\right)$, average- $\left(\mathrm{ID}_{50}=25-250\right)$, high$\left(I D_{50}=250-2500\right)$, and elite-neutralizers $\left(I_{50}>2500\right.$; Fig. 2a). Mean serum $I D_{50}$ titer was 111.3 with $17.7 \%$ of individuals that did not reach $50 \%$ neutralization at the lowest serum dilution of $1: 10$. In addition, all samples were purified for IgG and the neutralizing response was determined and categorized based on $I_{50}$ values into non- $\left(I_{50}>750 \mu \mathrm{g} / \mathrm{ml}\right)$, low- $\left(I C_{50}=500-750 \mu \mathrm{g} / \mathrm{ml}\right)$, average- $\left(I_{50}=100-500\right.$ $\mu \mathrm{g} / \mathrm{ml})$, high- $\left(\mathrm{IC}_{50}=20-100 \mu \mathrm{g} / \mathrm{ml}\right)$, and elite-neutralization $\left(\mathrm{IC}_{50}<20 \mu \mathrm{g} / \mathrm{ml} ;\right.$ Fig. 2b). At study visit 1 , out of 963 participants, $10 \%, 44.8 \%$, and $20 \%$ demonstrated low, average, and high neutralization, respectively. $21 \%$ did not mount an IgG neutralizing response of an $\mathrm{IC}_{50}$ below $750 \mu \mathrm{g} / \mathrm{ml}$. $3.3 \%$ of individuals were classified as 'elite neutralizers' with $\mathrm{IC}_{50}$ values as low as $0.7 \mu \mathrm{g} / \mathrm{ml}$ detected in one individual at 8.6 weeks post disease onset. Combining serum and IgG measurements, $87.3 \%$ individuals showed detectable NAb activity at median 7.3 weeks after SARS-CoV-2 infection (Fig. 2c). The serum and IgG neutralization potency categorization matched for most individuals with a high correlation between serum ID $I_{50}$ titers and IgG IC 50 values (spearman $r=-0.72, p<0.0001 ;$ Fig. 2 c). Moreover, only $5 \%$ samples had only serum and no IgG neutralization indicating that IgG antibodies forms the dominant NAb isotype in serum. To further determine the predictive value of IgG binding to the S protein for SARS-CoV-2 neutralization, we performed an S1-reactive ELISA (Euroimmun) on all samples of visit 1. $82.8 \%$ and $70.2 \%$ of individuals possessed spike-reactive IgG (Fig. 2 d, e) and IgA Abs, respectively (Fig. $\mathbf{2 d}$ and Extended Data Fig. 2a). Anti-spike IgG levels were directly proportional to IgG NAb IC 50 values (spearman $r=-0.62, p<0.0001$; Fig. 2f) and IgG Ab levels better correlated with serum neutralization than IgA Ab levels (Extended Data Fig. 2c, d).

Finally, we determined the fraction of individuals lacking any detectable antibody response. To this end, we combined the results of different IgG and IgA assays detecting binding to SARS-CoV-2 S1, S1/S2, and $\mathrm{N}$ as well as three neutralization assays (Fig. 2 g). Out of the 166 anti-S1-IgG negative (12.7\%) or equivocal (4.6\%) individuals, we found binding antibodies in $62.0 \%$ in at least one of four assays and neutralizing activity in $54.2 \%$ in at least one of three assays (Fig. $\mathbf{2 g}$, h). Combining these results and accounting for assay-specificity (see methods) we show that only $5.6 \%-7.3 \%$ of individuals do not mount a detectable antibody response against SARS-CoV-2. Notably, while only 3.6\% (1 of 28) of hospitalized patients and $4.9 \%$ (43 of 877 ) of individuals with mild symptoms lacked anti-SARS-CoV-2 antibodies, 
22.7\% (10 of 44) asymptomatic individuals were negative for a detectable antibody response in at visit 1. We conclude that $92.7-94.4 \%$ of individuals naturally infected with SARS-CoV-2 mount an antibody response against the virus within the first 12 weeks. Among those, we detected a broad variation in neutralizing activity with approximately $3 \%$ generating a highly potent serum and IgG NAb response.

\section{Sero-reactivity, age, and disease severity predict SARS-CoV-2 neutralization}

Next, we analyzed how age, disease severity, gender, and the presence of pre-existing conditions correlate with the anti-spike antibody and SARS-CoV-2 neutralizing response (Fig. 3a, Extended Data Fig. 3). The IgG NAb response was significantly higher in older individuals $(p<0.0001)$, with participants $>60$ years comprising $7.7 \%$ of elite- and $42.8 \%$ of high-neutralizers (Fig. $3 a$ ). Hospitalized patients and individuals with symptoms had significantly higher NAb activity $(p=0.0008$ and 0.0003$)$ compared to asymptomatic individuals, of which $43.2 \%$ (25 of 44) lacked detectable IgG NAbs (Fig. 3a). Males showed higher SARSCoV-2 neutralization than females (GeoMean $\mathrm{IC}_{50} 136.3 \mu \mathrm{g} / \mathrm{ml}$ vs. $188.4 \mu \mathrm{g} / \mathrm{ml} ; \mathrm{p}<0.0001$ ). In addition, individuals with pre-existing conditions had slightly higher NAb activity compared to those without them (GeoMean IC $50161.9 \mu \mathrm{g} / \mathrm{ml}$ vs. $174.6 \mu \mathrm{g} / \mathrm{ml} ; \mathrm{p}=0.022$; Fig. 3a). Similar to IgG NAb activity, serum neutralizing activity and anti-spike antibodies were also higher in older individuals, patients with a more severe course of disease, and males (Extended Data Fig. 3a-c). Next, we performed a multivariate statistical analysis to determine the interplay between clinical features and the NAb response. Features included gender, age, disease severity, presence of pre-existing conditions, disease symptoms (Supplementary Table 1), weeks since disease onset, and the anti-spike IgG/IgA ELISA measurements. We applied a stepwise regression that adds new features only if they significantly improved the model according to a likelihood ratio test. The resulting $\mathrm{IC}_{50}$ prediction model (Adjusted $\mathrm{R}^{2}=0.461$ ) revealed that $\lg$ antibody levels are most predictive for SARS-CoV-2 neutralizing activity $\left(p=10^{-99}\right)$, followed by age $\left(p=6.1 * 10^{-7}\right), \lg A$ antibody levels $\left(p=7.6^{*} 10^{-6}\right)$, time since disease onset $(p=0.01)$ and fever during infection ( $p=0.02$; Fig. 3b, c). Similarly, age, anti-spike antibody levels, times since disease onset and fever during acute infection were also found to be highly predictive of serum ID ${ }_{50}$ (Extended Data Fig. 4a, 4b). Additionally, we built a Bayesian network model to determine the feature dependencies and how they predict the SARS-CoV-2 IgG neutralizing response (Fig. 3d). When applying the stepwise regression model only for predicting the presence of anti-spike antibodies, we observed that gender ( $\operatorname{lgG} p=8.5^{\star} 10^{-5}$; $\operatorname{lgA} p$ $\left.=2.2 * 10^{-10}\right)$ and the disease symptoms, cough $(\operatorname{IgA} p=0.01)$, diarrhea $(\operatorname{lgG} p=0.02)$ or change in taste $(\lg \mathrm{p}=0.002 ; \lg A \mathrm{p}=0.04)$ are predictive of anti-spike antibody levels (Extended Data Fig. 4b, c). In addition, we investigated the possible effect of viral load obtained from naso-/oro-pharyngeal swabs at the time of diagnosis on the antibody response at study visit 1, but no correlation was found (Extended Data Fig. 4d, e). In summary, higher IgG levels, older age and fever during acute infection are highly predictive of the development of SARS-CoV-2 neutralizing activity.

\section{Elite SARS-CoV-2 neutralizers exhibit SARS-CoV-1 cross-neutralization}

Individuals mounting a highly potent neutralizing antibody response are often considered 'elite neutralizers'38. These individuals are of particular interest i.) to identify factors associated with the development of effective humoral immunity, ii.) to guide vaccine design, and iii.) to isolate highly potent 
neutralizing monoclonal antibodies ${ }^{39}$. In order to characterize the small fraction of SARS-CoV-2 elite neutralizers in our cohort ( $3 \% ; I_{50}<20 \mu \mathrm{g} / \mathrm{ml}$; Fig. 2b), we selected 15 individuals of each group of non, low, average, high and elite-neutralizers (Extended Data Fig. 5a-c) testing them against authentic SARSCoV-2 as well as SARS-CoV-1 pseudovirus. Neutralization of SARS-CoV-2 pseudovirus against authentic virus correlated closely in all groups with authentic virus (spearman $r=0.79$; Extended data Fig. $5 d$ ). SARS-CoV-1 neutralization was not observed in non- and low-neutralizers and only in 1 out of 15 average neutralizers. However, in the high and elite neutralizing groups, 8/15 and 15/15 samples carried SARSCoV-1 cross-neutralizing activity, respectively, with potencies $\left(\mathrm{IC}_{50}\right)$ as low as $5.1 \mu \mathrm{g} / \mathrm{ml} \mathrm{lgG}$. Of note, while all SARS-CoV-2 elite neutralizers demonstrated SARS-CoV-1 cross-neutralization, variation in potency is ranging from $12.1-634.9 \mu \mathrm{g} / \mathrm{ml}$ and an overall low correlation (spearman $r=0.3745$; Fig. 4b). Next, we studied the neutralizing potency of the elite neutralizers against six different SARS-CoV-2 strains carrying several mutations that became prominent at a global level ${ }^{40}$ (Fig. 4c, Extended Data Fig. 5). IgG from elite neutralizers was potent against all tested SARS-CoV-2 strains including both S1 and S2 mutants as well as variants (BAVP1, DRC94) carrying the D614G mutation (Fig. 4c, Extended Data Fig. 5). We conclude, that individuals mounting a potent SARS-CoV-2 NAb response possess cross-reactive antibodies against SARS-CoV-1 without any known prior exposure and are effective in neutralizing various prevalent SARSCoV-2 strains.

\section{Long-term persistence of IgG NAbs after SARS-CoV-2 infection}

In order to study antibody kinetics, we first investigated the development of SARS-CoV-2-directed antibodies in the first 4 weeks after disease onset. To this end, we evaluated 259 samples obtained from an additional 110 individuals. In this subgroup, $44.5 \%$ and $54,5 \%$ were male and female, respectively, and 41.8\% had been hospitalized (Extended Data Fig. 6a). Anti-spike IgG and IgA could be detected in some people within the first week after disease onset, with IgA levels starting to decline by week 4 (Extended Data Fig. 6b). Out of the 24 individuals that were closely monitored, most individuals sero-converted between 2-3 weeks post disease onset (Extended Data Fig. 6b).

In order to assess longevity of humoral immunity following SARS-CoV-2 infection, we applied a linear regression mixed effects model to antibody measurements obtained between 3.1 to 41.9 weeks post infection. The half-life of anti-spike IgG was estimated to be 34.9 weeks (Fig. 5a). For systematic tracking of the antibody response within individuals, we analyzed anti-spike antibodies in 131 individuals at 4 study visits (range 3.1 to 38.7 weeks post disease onset; Fig. 5b, c). The data show that IgG levels decrease between 1 st to 2 nd study visit (Geo. Mean $S / C 0=4.6$ vs. Geo. Mean $S / C 0=3.7$ ) followed by a relatively constant IgG levels for 10 months after infection (Geo. Mean $\mathrm{S} / \mathrm{CO}=3.0$ ) (Fig. 5b,

Supplementary Table 1). While the detection of S1-reactivity stays equal at first and second visit (86\%), the fraction of individuals that are reactive for S1-reactive antibodies decays to $79 \%$ ( $7 \%$ drop from visit 1 ) at the third visit and to $73 \%$ (13\% drop from visit 1) at visit 4 (9-10 months post disease onset).

NAb activity was longitudinally monitored for 342 individuals from visit 1 (median 6.4 weeks post infection) to visit 2 (median 17.3 weeks post infection) (Fig. $\mathbf{5 d - g}$ ). Regression modeling showed that serum NAb titers had a short half-life of 6.7 weeks compared to a much longer 30.8-week half-life for IgG 
NAb titers (Fig. 5 d, e). Out of 342 individuals, $87.1 \%$ had serum NAb activity at visit 1 whereas only $70.5 \%$ had NAb activity remaining at visit 2 (Fig. 5f). The overall fraction of IgG neutralizers changed from 82$75 \%$ between visit 1 and 2. The most dramatic drop from Geo Mean $I_{50}$ of $16.23 \mu \mathrm{g} / \mathrm{ml}$ to $45.54 \mu \mathrm{g} / \mathrm{ml}$ was detected in elite neutralizers, $88 \%$ of whom lost their 'elite' status. $23.9 \%$ of average/low neutralizers at visit 1 became negative at visit 2 (Fig. $\mathbf{5 g}$ ). Approximately $11 \%$ of individuals did not develop any NAbs and remained serum and IgG-negative at both visits. Overall, only $2.4 \%$ of the cohort lost detectable antibody responses against SARS-CoV-2 between 1.5 and 4.5 months post infection (Extended data

Fig. 7a-e).

In summary, these results show that in most individuals anti-spike lgG antibody levels are maintained for 10 months with a half-life estimate of 8.7 months. Moreover, even though there is a rapid decline in serum NAb activity, IgG NAb function remains relatively constant with an estimated half-life of 7.7 months. We conclude that although there is a decay of antibody titers in serum, the humoral IgG response persists for as long as 10 months after SARS-CoV-2 infection.

\section{Discussion}

In order to end the COVID-19 pandemic, widespread SARS-CoV-2 protective immunity will be required. Antibodies are critical for effective clearance of pathogens and for prevention of viral infections ${ }^{41}$. In this study, we examined the neutralizing antibody response in 963 individuals who had recently recovered from SARS-CoV-2 infection. The cohort consists primarily (91.69\%) of patients with mild COVID-19 therefore representing the predominant clinical course of this disease ${ }^{6}$. Since higher disease severity was shown to correlate with higher antibody responses ${ }^{14,42}$, cohorts mainly composed of hospitalized individuals have limited applicability on the majority of COVID-19 cases $20,35,43,44$. Moreover, to our knowledge this is the most comprehensive study $(n=963)$, in which neutralizing antibody activity has been reported to date with the next largest study having analyzed $4-5$ fold less individuals at a single time point ${ }^{45}$.

Upon recovery from COVID-19, we detected the development of a broad spectrum of IgG neutralizing activity ranging from no neutralization (threshold $\mathrm{IC}_{50}<750 \mu \mathrm{g} / \mathrm{ml}, 21 \%$ ) to low $\left(\mathrm{IC}_{50}=50-750 \mu \mathrm{g} / \mathrm{ml}\right.$, $10 \%)$, average $\left(\mathrm{IC}_{50}=100-500 \mu \mathrm{g} / \mathrm{ml}, 44.8 \%\right)$, high $\left(\mathrm{IC}_{50}=20-100 \mu \mathrm{g} / \mathrm{ml}, 20.9 \%\right)$, and elite SARS-CoV-2 neutralization ( $\mathrm{IC}_{50}<20 \mu \mathrm{g} / \mathrm{ml}, 3.3 \%$ ). $94.4 \%$ of individuals were found to possess $\mathrm{S}$ - or N-reactive antibodies or neutralizing activity at serum or IgG level. Thus, while most individuals develop a detectable antibody response upon natural infection, the extent of SARS-CoV-2 neutralizing activity is highly variable with the fraction of non-responders being highest for asymptomatic individuals (23\%).

The broad spectrum of neutralizing activity developed in COVID-19 recovered individuals may impact the level of protective immunity. For instance, asymptomatic infection is estimated to account for up to $40 \%$ of all infections ${ }^{46}$. In these individuals and in other patients with weak antibody responses, lower lgG titers may contribute to a higher susceptibility to re-infection. Recently, mutated virus strains were 
reported 47,48 , some of which possess mutations causing partial resistance to convalescent plasma ${ }^{48}$ or SARS-CoV-2 monoclonal antibodies ${ }^{49}$. A weak antibody response may help propagate escape variants and may therefore complicate effective measures to combat the COVID-19 pandemic.

To guide vaccine strategies based on population demographics, it is critical to understand which clinical features affect the development of antibody responses. NAb response presented here is comparable to recent spike-based mRNA vaccine studies in age group 18-55, where geometric mean neutralizing titers were in the range of $100-300 I_{50}$ (depending on dose) 1.5 months post vaccination ${ }^{17,50}$ versus 111.3 $I_{50}$ in this study. Recent studies have reported that age, gender and disease severity ${ }^{14,36,44}$ can impact SARS-CoV-2 NAb titers ${ }^{14,36,42,43,45}$. However, a comprehensive analysis on a large representative cohort was missing. Using multivariate statistical analysis on the antibody measurements and clinical data, we found that higher anti-spike antibody levels, older age, symptomatic infection and a severe course of COVID-19 were highly predictive of NAb titers. Notably, based on previous vaccine studies ${ }^{51}$, it was frequently speculated that older individuals might generate a less efficient immune responses to SARSCoV-2 infection or vaccination. However, based on our data, the $>60$ age group had the highest level of neutralizing $\mathrm{IgG}$ antibodies (mean $\mathrm{IC}_{50}=84.8 \mu \mathrm{g} / \mathrm{ml}$, mean $\mathrm{ID}_{50}$ serum titer $=276.6$ ).

In some individuals we detected very high levels of SARS-CoV-2 neutralizing activity $\left(\mathrm{IC}_{50}<20 \mu \mathrm{g} / \mathrm{ml}, \mathrm{ID}_{50}\right.$ serum titer $>2,500$ ) ranking them as 'elite neutralizers'. While cross-reactivity against SARS-CoV-1 and other Beta-CoVs has been shown for some SARS-CoV-2 recovered individuals ${ }^{52-54}$, we revealed that all elite neutralizers have cross-reactive IgG NAbs against SARS-CoV-1. Moreover, IgG from elite neutralizers could efficiently block infection of 6 SARS-CoV-2 strains. Two of them (BavP1 and DRC94) contain the D614G mutation in the S protein ${ }^{55}$ associated with higher infectivity ${ }^{56}$. Given the eminent risk of novel emerging CoVs and monoclonal antibody-resistant SARS-CoV-2 variants, developing antibodies with broader neutralization breadth would be critical. Further evaluation of the antibody response in such elite neutralizers at the single B cell level will be required to understand the details of such potent NAb responses and can yield the identification of new highly potent cross-reactive monoclonal antibodies.

Effective neutralization and clearance of pathogens is mainly mediated by IgG antibodies, which are typically formed within 1-3 weeks post infection and often provide long-term immunity that can last decades $^{57}$. Protective immunity to seasonal coronaviruses like NL63, 229E, OC43 and HKU1 is known to be short lived and re-infection is common ${ }^{58}$. In addition, the antibody response to SARS-CoV- 1 and Middle East Respiratory Syndrome (MERS)-CoV was shown to wane over time ${ }^{59}$. Upon SARS-CoV-1 infection, serum IgG and NAbs were shown to decline 3 years after infection ${ }^{60}$. This suggests that immunity to CoVs is rather short lived compared to some other viruses such as measles virus, for which life-long antibody immunity is observed ${ }^{57}$. In our study we not only measured serum neutralization, but also quantified SARS-CoV-2 IgG neutralizing activity. While serum neutralization waned quickly (half-life of 1.5 months), levels of purified IgG rather persisted with a longer half-life of 7.7 months. The sharp drop in serum neutralization could be a consequence of a decline in anti-spike $\lg A$ and $\lg M$ titers $^{34}$, which along 
with IgG, cumulatively contribute to serum NAb activity ${ }^{61}$. Finally, SARS-CoV-2 spike-based mRNA vaccines ${ }^{17}$ were shown to induce NAb titers in different age groups for a time span up to 4.25 months ${ }^{18}$. In this study, we found that although SARS-CoV-2-reactive IgG levels decline by $17 \%$ within the first 4 months after infection, anti-spike IgG can be persistently detected in the majority of COVID-19 cases for up to 10 months post infection.

In summary, the data presented in this study provides new insight into the features that shape the SARSCoV-2 NAb response in COVID-19 recovered individuals. Longitudinal mapping of antibody responses reveals a relatively long-lived IgG antibody response lasting up to 10 months. Since many SARS-CoV-2 vaccines are spike protein-based ${ }^{62}$, studying antibody dynamics informs us on longevity of natural immunity as well as may help to inform on vaccination strategies and outcomes in the population.

\section{Methods}

\section{Enrollment of participants and study design}

Blood samples were collected from donors who gave their written consent under the protocols 20-1187 and 16-054, approved by the Institutional Review Board (IRB) of the University Hospital Cologne. All samples were handled according to the safety guidelines of the University Hospital Cologne. Individuals that met the inclusion criteria of i.) $\geq 18$ years old and ii.) history of SARS-CoV-2 positive polymerase chain reaction (PCR) from nasopharyngeal swab or collected sputum, and/or iii.) an onset of COVID-19 specific symptoms longer than 3 weeks ago, were enrolled in this study. Demographical data, COVID-19related pre-existing conditions, and information on the clinical course were collected at study visit 1. Blood samples were collected starting from study visit 1, for up to 4 follow up visits between the 6th of April and 17th of December 2020.

\section{Processing of serum, plasma and whole blood samples}

Blood samples were collected in Heparin syringes or EDTA monovette tubes (Becton Dickinson) and fractionated into plasma and peripheral blood mononuclear cell (PBMC) by density gradient centrifugation using Histopaque-1077 (Sigma). Plasma aliquots were stored at $-80^{\circ} \mathrm{C}$ till use. Serum was collected from Serum-gel tubes (Sarstedt) by centrifugation and stored at $-80^{\circ} \mathrm{C}$ till use.

\section{Isolation of IgGs from serum and plasma samples}

For the isolation of total IgG, $0.5-1 \mathrm{~mL}$ plasma or serum was heat inactivated at $56^{\circ} \mathrm{C}$ for 45 minutes and incubated overnight with Protein G Sepharose 4 Fast Flow beads (GE Healthcare) at $4{ }^{\circ} \mathrm{C}$. Next day, beads were washed on chromatography columns (BioRad) and Protein G bound IgG was eluted using $0.1 \mathrm{M}$ Glycine $\mathrm{pH}=3$ and instantly buffered in $1 \mathrm{M}$ Tris $\mathrm{pH}=8$. Buffer exchange to PBS (Gibco) was performed using $30 \mathrm{kDa}$ Amicon Ultra-15 columns (Millipore) and the purified IgG was stored at $4{ }^{\circ} \mathrm{C}$.

\section{Cloning of SARS-CoV-2 spike variants}

The codon optimized SARS-CoV-2 Wu01 spike (EPI_ISL_40671) was cloned into pCDNA ${ }^{\mathrm{TM}}$ 3.1/V5HisTOPO vector (Invitrogen). SARS-2-S global strains (BavP1 EPI_ISL_406862; ARA36 EPI_ISL_418432; 
DRC94 EPI_ISL_417947; CA5 EPI_ISL_408010; NRW8 EPI_ISL_414508) were generated by introducing the corresponding amino acid mutations (Extended Data Fig. 5) using the Q5® Site-Directed Mutagenesis Kit (NEB) and per manufacturer's protocol.

\section{Production of SARS-CoV pseudovirus particles}

Pseudovirus particles were generated by co-transfection of individual plasmids encoding HIV-1 Tat, HIV-1 Gag/Pol, HIV-1 Rev, luciferase followed by an IRES and ZsGreen, and the SARS-CoV-2 spike protein as previously described $^{63}$. In brief, HEK 293T cells were transfected with the pseudovirus encoding plasmids using FuGENE 6 Transfection Reagent (Promega). The virus culture supernatant was harvested at $48 \mathrm{~h}$ and $72 \mathrm{~h}$ post transfection and stored at $-80{ }^{\circ} \mathrm{C}$ until use. Each virus batch was titrated by infecting $293 \mathrm{~T}-$ ACE2 and after a 48-hour incubation period at $37^{\circ} \mathrm{C}$ and $5 \% \mathrm{CO}_{2}$, luciferase activity was determined after addition of luciferin/lysis buffer (10 mM MgCl2, 0.3 mM ATP, 0.5 mM Coenzyme A, 17 mM IGEPAL (all Sigma-Aldrich), and $1 \mathrm{mM}$ D-Luciferin (GoldBio) in Tris-HCL) using a microplate reader (Berthold). An RLU of approximately 1000-fold in infected cells versus non-infected cells was used for neutralization assays.

\section{Pseudovirus assay to determine IgG/plasma/serum SARS-CoV-2 neutralizing activity}

For testing SARS-CoV-2 neutralizing activity of IgG or serum/plasma samples, serial dilutions of IgG or serum/plasma (heat inactivated at $56^{\circ} \mathrm{C}$ for $45 \mathrm{~min}$ ) were co-incubated with pseudovirus supernatants for $1 \mathrm{~h}$ at $37^{\circ} \mathrm{C}$ prior to addition of 293T cells engineered to express ACE2 ${ }^{63}$. Following a 48-hour incubation at $37^{\circ} \mathrm{C}$ and $5 \% \mathrm{CO}_{2}$, luciferase activity was determined using the reagents described above. After subtracting background relative luminescence units (RLUs) of non-infected cells, $50 \%$ inhibitory concentrations (IC50s) were determined as the IgG concentrations resulting in a 50\% RLU reduction compared to untreated virus control wells. $50 \%$ Inhibitory dose $\left(\right.$ ID $\left._{50}\right)$ was determined as the serum dilution resulting in a 50\% reduction in RLU compared to the untreated virus control wells. Each IgG and serum sample were measured in two independent experiments on different days and the average $I_{50}$ or $\mathrm{ID}_{50}$ values have been reported. For each run, a SARS-CoV-2 neutralizing monoclonal antibody was used as control to ensure consistent reproducibility in experiments carried out on different days. Assay specificity calculated using pre-COVID-19 samples was found to be $100 \% . I_{50}$ and ID I0 $_{50}$ values were calculated in GraphPad Prism 7.0 by plotting a dose response curve.

\section{SARS-CoV-2 live virus isolation from nasopharyngeal swabs}

For outgrowth cultures of authentic SARS-CoV-2 from nasopharyngeal swabs, $1 \times 10^{6}$ VeroE6 cells were seeded onto a T25 flask (Sarstedt) on the previous day DMEM (Gibco) containing 10\% FBS, $1 \%$ PS, 1 mM L-Glutamine and $1 \mathrm{mM}$ Sodium pyruvate. $0.2 \mathrm{~mL}$ swab in VNT medium was diluted with 0.8 mL DMEM (Gibco) containing 2\% FBS, 1\% PS, $1 \mathrm{mM}$ L-Glutamine and $1 \mathrm{mM}$ Sodium pyruvate. The swab dilution was added to VeroE6 cells and left for 1 hour at $37^{\circ} \mathrm{C}, 5 \% \mathrm{CO}_{2}$ after which an additional $3 \mathrm{~mL}$ medium was added. The cultures were examined for the next days for CPE and samples were sent for viral load analysis to track growth of virus by E-gene qPCR. Cell culture supernatant was harvested from positive cultures and stored at $-150{ }^{\circ} \mathrm{C}$ until use. Virus was titrated by adding serial dilutions of virus supernatant (8 replicates) on VeroE6 cells in DMEM (Gibco) containing 2\% FBS, 1\% PS, $1 \mathrm{mM}$ L-Glutamine and $1 \mathrm{mM}$ 
Sodium pyruvate. After 4 days of incubation at $37{ }^{\circ} \mathrm{C}, 5 \% \mathrm{CO}_{2}$, the presence or absence of cytopathic effects (CPE) was noted in using a brightfield microscope. $\mathrm{TCID}_{50}$ was calculated using the Spearman and Kaerber algorithm 64,65 .

\section{SARS-CoV-2 live virus neutralization assay}

Live SARS-CoV-2 (termed CoV2-P3) was grown out from a swab from Cologne using VeroE6 cells as described above and then expanded in culture by superinfection of VeroE6 from the initial outgrowth culture. Whole genome sequencing of the isolated virus was done isolating viral RNA using the QIAamp MinElute Virus Spine kit (Qiagen) and performing Illumina sequencing. The virus spike amino acid sequence (Extended Data Fig. 5) is similar to the Wu01 spike (EPI_ISL_40671) with the exception that it contains the D641G mutation. For the neutralization assay, dilutions of IgG were co-incubated with the virus $\left(1000-2000 \mathrm{TCID}_{50}\right)$ for $1 \mathrm{~h}$ at $37^{\circ} \mathrm{C}$ prior to addition of VeroE6 cells in DMEM (Gibco) containing $2 \%$ FBS, $1 \%$ PS, $1 \mathrm{mM} \mathrm{L-Glutamine}$ and $1 \mathrm{mM}$ Sodium pyruvate. After 4 days of incubation at $37^{\circ} \mathrm{C}, 5 \%$ $\mathrm{CO}_{2}$, neutralization was analyzed by observing cytopathic effects (CPE) using a brightfield microscope and the highest dilution well with no $\mathrm{CPE}$ was noted to be the $\mathrm{IC}_{100}$ for the antibody. Assay specificity calculated using pre-COVID-19 samples was found to be $100 \%$. All samples were measured in two independent experiments on separate days and the average $\mathrm{IC}_{100}$ from all measurements has been reported.

\section{Detection of anti-SARS-CoV-2 spike IgG and IgA by ELISA}

For assessing IgA and IgG antibody titers, the Euroimmun anti-SARS-CoV-2 ELISA using the S1 domain of the spike protein as antigen was used (Euroimmun Diagnostik, Lübeck, Germany). Serum or plasma samples were tested on the automated system Euroimmun Analyzer I according to manufacturer's recommendations. Signal-to-cut-off $(\mathrm{S} / \mathrm{CO})$ ratio was calculated as extinction value of patient sample/extinction value of calibrator. $\lg A$ and $\lg G S / C O$ values were interpreted as positive $S / C O \geq 1.1$, equivocal $\mathrm{S} / \mathrm{CO} \geq 0.8-<1.1$, and negative $\mathrm{S} / \mathrm{CO}<0.8$. Additional commercial kits used for antibody measurements were also used as per manufacturer's recommendations; Anti-S1/S2 IgG was measured using DiaSorin's LIAISON® SARS-CoV-2 ELISA kit with the following cut-off values: negative $<12.0 \mathrm{AU} / \mathrm{ml}$, equivocal $\geq 12.0-<15.0 \mathrm{AU} / \mathrm{ml}$ and positive $\geq 15.0 \mathrm{AU} / \mathrm{ml}$. Anti-N Pan-lgs were measured using Roche's Elecsys ${ }^{\circledR}$-Assay with cut-off values: non-reactive $<1,0 \mathrm{COI}$ and reactive $\geq 1,0 \mathrm{COI}$. Anti-N IgG were measured with Abbott's Alinity i system with cut-off values: positive $S / C O \geq 1.4$ and negative $S / C O<1.4$. Assay specificities calculated using pre-COVID-19 samples: Euroimmun IgG 100\%; Euroimmun IgA 96\%; Roche 98\%; Diasorin 98\%; Abbott 98\%.

\section{Measurement of SARS-CoV-2 RNA levels from nasopharyngeal swabs}

Cycle threshold values for quantifying viral load in naso/oro-pharyngeal swabs was done by qPCR using LightMix ${ }^{\circledR}$ SarbecoV E-gene ${ }^{66}$ plus EAV control (TIB Molbiol, Berlin, Germany) in combination with the Ngene (inhouse primer sets in multiplex PCR) on LightCycler® 480 (Roche Diagnostics).

\section{Statistical modeling}


To select features that are predictive for the $\log _{10}$ response in a multivariate analysis (Fig. 3b), forward stepwise regression was applied, using the $\mathrm{p}$-value from a likelihood ratio test (R function Imtest::Irtest) as selection criterion in each step. The final multiple linear regression model (Fig. 3c) includes only features that show a significant model improvement $(a l p h a=0.05)$ in the feature selection phase. To study the interplay of the different features regarding their relationship with the response (Fig. 3d), a Bayesian network was learned by maximizing the BIC score for hybrid networks via hill-climbing $(R$ function bnlearn::hc $)^{67}$. To enforce it to be a sink in the network, all outgoing edges from the response variable were blacklisted prior to learning. For the longitudinal analyses (Fig. 5e-h), linear mixed effect models (R-function nlme:Ime) were applied to all data points from both visits, where each patient has its own intercept. Since a binary transformation of the response was used, half-life estimates were computed as negative inverse of the common slope regression coefficient. Prediction intervals were computed using R-function ggeffects::.ggpredict ${ }^{68}$.

\section{Declarations}

\section{Data availability statement}

All data including virus spike sequences are available in the manuscript main figures or supplementary material.

\section{Acknowledgements}

We are extremely grateful to all study participants who took part in this study; members of the Klein lab for helpful discussions; Reinhild Brinker, Marie Wunsch and Maike Wirtz for technical support; Daniela Weiland and Nadine Henn for project and laboratory management support; Stefan Poehlmann and Markus Hoffmann for sharing the Wuhan SARS-2-S spike construct; Jesse Bloom and Kate Crawford for sharing 293T-ACE2 cells and lentiviral constructs for production of SARS-CoV pseudovirus particles; Jason McLellan and Nianshuang Wang for sharing the SARS-1-S spike construct; Stephan Becker and Verena Kraehliv for sharing VeroE6 cells; Jeorg Timm, Andreas Walker and Max Damagnez for SARSCoV-2 virus genome sequencing. This work was funded by grants to Florian Klein from the German Center for Infection Research (DZIF), the German Research Foundation (DFG) CRC1279 and CRC1310, European Research Council (ERC) ERC-stG639961 and COVIM: „NaFoUniMedCovid19“ (FKZ: 01KX2021).

\section{Author contributions}

F.Klein and K.V. conceptualized and designed the study; F.Klein, C.L., G.F. and N.P. provided supervision; K.V. and F.Klein wrote the first draft of the manuscript, all authors reviewed the manuscript draft and agree to the final version; M.A., P.S., L.G., F.D., V.C., H.G., C.H., I.S., N.J., were involved in study participant interaction including obtaining informed consent, clinical data and sample collection and writing the study protocol. K.V. and F.Kleipass performed neutralization assays; V.C. and W.J. obtained ELISA data; F.Kleipass and K.V. performed IgG purification; K.V., V.C., F.K., F.D., P.S. analyzed data; K.V. performed final 
data analysis and R.E. performed statistical analysis; M.S., M.S.E., R.S. and P.M. processed blood samples; K.E., S.S. and E.H. were involved in data collection.

\section{References}

1 Zhu, N. et al. A Novel Coronavirus from Patients with Pneumonia in China, 2019. N Engl J Med 382, 727-733, doi:10.1056/NEJMoa2001017 (2020).

2 Zhou, P. et al. A pneumonia outbreak associated with a new coronavirus of probable bat origin. Nature 579, 270-273, doi:10.1038/s41586-020-2012-7 (2020).

3 Huang, C. et al. Clinical features of patients infected with 2019 novel coronavirus in Wuhan, China. The Lancet 395, 497-506, doi:10.1016/s0140-6736(20)30183-5 (2020).

4 Mizrahi, B. et al. Longitudinal symptom dynamics of COVID-19 infection. Nat Commun 11, 6208, doi:10.1038/s41467-020-20053-y (2020).

5 Rothe, C. et al. Transmission of 2019-nCoV Infection from an Asymptomatic Contact in Germany. N Engl J Med 382, 970-971, doi:10.1056/NEJMc2001468 (2020).

6 Williamson, E. J. et al. Factors associated with COVID-19-related death using OpenSAFELY. Nature 584, 430-436, doi:10.1038/s41586-020-2521-4 (2020).

$7 \quad$ Wolfel, R. et al. Virological assessment of hospitalized patients with COVID-2019. Nature 581, 465469, doi:10.1038/s41586-020-2196-x (2020).

8 He, X. et al. Temporal dynamics in viral shedding and transmissibility of COVID-19. Nat Med 26, 672-675, doi:10.1038/s41591-020-0869-5 (2020).

9 Galvan-Tejada, C. E. et al. Persistence of COVID-19 Symptoms after Recovery in Mexican Population. Int J Environ Res Public Health 17, doi:10.3390/ijerph17249367 (2020).

10 Cirulli, E. T. et al. Long-term COVID-19 symptoms in a large unselected population. doi:10.1101/2020.10.07.20208702 (2020).

11 Huang. 6-month consequences of COVID-19 in patients discharged from hospital: a cohort study. Lancet, doi:https://doi.org/10.1016/S0140-6736(20)32656-8 (2021).

12 Walls, A. C. et al. Structure, Function, and Antigenicity of the SARS-CoV-2 Spike Glycoprotein. Cell 181, 281-292 e286, doi:10.1016/j.cell.2020.02.058 (2020).

13 Hoffmann, M. et al. SARS-CoV-2 Cell Entry Depends on ACE2 and TMPRSS2 and Is Blocked by a Clinically Proven Protease Inhibitor. Cell, doi:10.1016/j.cell.2020.02.052 (2020). 
14 Piccoli, L. et al. Mapping Neutralizing and Immunodominant Sites on the SARS-CoV-2 Spike Receptor-Binding Domain by Structure-Guided High-Resolution Serology. Cell, doi:10.1016/j.cell.2020.09.037 (2020).

15 Rydyznski Moderbacher, C. et al. Antigen-Specific Adaptive Immunity to SARS-CoV-2 in Acute COVID-19 and Associations with Age and Disease Severity. Cell 183, 996-1012 e1019, doi:10.1016/j.cell.2020.09.038 (2020).

$16 \mathrm{Ni}$, L. et al. Detection of SARS-CoV-2-Specific Humoral and Cellular Immunity in COVID-19 Convalescent Individuals. Immunity 52, 971-977 e973, doi:10.1016/j.immuni.2020.04.023 (2020).

17 Sahin, U. et al. COVID-19 vaccine BNT162b1 elicits human antibody and TH1 T cell responses. Nature 586, 594-599, doi:10.1038/s41586-020-2814-7 (2020).

18 Widge, A. T. et al. Durability of Responses after SARS-CoV-2 mRNA-1273 Vaccination. N Engl J Med, doi:10.1056/NEJMc2032195 (2020).

19 Long, Q. X. et al. Antibody responses to SARS-CoV-2 in patients with COVID-19. Nat Med 26, 845848, doi:10.1038/s41591-020-0897-1 (2020).

20 Seow, J. et al. Longitudinal observation and decline of neutralizing antibody responses in the three months following SARS-CoV-2 infection in humans. Nat Microbio/ 5, 1598-1607, doi:10.1038/s41564020-00813-8 (2020).

21 Wajnberg, A. et al. Robust neutralizing antibodies to SARS-CoV-2 infection persist for months. Science, doi:10.1126/science.abd7728 (2020).

22 Zohar, T. \& Alter, G. Dissecting antibody-mediated protection against SARS-CoV-2. Nat Rev Immunol 20, 392-394, doi:10.1038/s41577-020-0359-5 (2020).

23 Dan, J. M. et al., doi:10.1101/2020.11.15.383323 (2020).

24 Corti, D. \& Lanzavecchia, A. Broadly neutralizing antiviral antibodies. Annu Rev Immuno/ 31, 705742, doi:10.1146/annurev-immunol-032712-095916 (2013).

25 Mercado, N. B. et al. Single-shot Ad26 vaccine protects against SARS-CoV-2 in rhesus macaques. Nature 586, 583-588, doi:10.1038/s41586-020-2607-z (2020).

26 McMahan, K. et al. Correlates of protection against SARS-CoV-2 in rhesus macaques. Nature, doi:10.1038/s41586-020-03041-6 (2020).

27 Zost, S. J. et al. Rapid isolation and profiling of a diverse panel of human monoclonal antibodies targeting the SARS-CoV-2 spike protein. Nat Med 26, 1422-1427, doi:10.1038/s41591-020-0998-x (2020). 
28 Kreer, C. et al. Longitudinal Isolation of Potent Near-Germline SARS-CoV-2-Neutralizing Antibodies from COVID-19 Patients. Cell 182, 843-854 e812, doi:10.1016/j.cell.2020.06.044 (2020).

29 Robbiani, D. F. et al. Convergent antibody responses to SARS-CoV-2 in convalescent individuals. Nature 584, 437-442, doi:10.1038/s41586-020-2456-9 (2020).

30 Chen, P. et al. SARS-CoV-2 Neutralizing Antibody LY-CoV555 in Outpatients with Covid-19. N Engl J Med, doi:10.1056/NEJMoa2029849 (2020).

31 Weinreich, D. M. et al. REGN-COV2, a Neutralizing Antibody Cocktail, in Outpatients with Covid-19. N Engl J Med, doi:10.1056/NEJMoa2035002 (2020).

$32 \mathrm{Ng}, \mathrm{D}$. L. et al. SARS-CoV-2 seroprevalence and neutralizing activity in donor and patient blood. Nat Commun 11, 4698, doi:10.1038/s41467-020-18468-8 (2020).

33 Wu, F. et al. Neutralizing antibody responses to SARS-CoV-2 in a COVID-19 recovered 2 patient cohort and their implications. MedRxiv, doi:10.1101/2020.03.30.20047365 (2020).

34 lyer, A. S. et al. Persistence and decay of human antibody responses to the receptor binding domain of SARS-CoV-2 spike protein in COVID-19 patients. Sci Immuno/ 5, doi:10.1126/sciimmunol.abe0367 (2020).

35 Zeng, C. et al. Neutralizing antibody against SARS-CoV-2 spike in COVID-19 patients, health care workers, and convalescent plasma donors. JCI Insight 5, doi:10.1172/jci.insight.143213 (2020).

36 Chen, X. et al. Disease severity dictates SARS-CoV-2-specific neutralizing antibody responses in COVID-19. Signal Transduct Target Ther 5, 180, doi:10.1038/s41392-020-00301-9 (2020).

37 Lanzavecchia, A., Fruhwirth, A., Perez, L. \& Corti, D. Antibody-guided vaccine design: identification of protective epitopes. Curr Opin Immunol 41, 62-67, doi:10.1016/j.coi.2016.06.001 (2016).

38 Simek, M. D. et al. Human immunodeficiency virus type 1 elite neutralizers: individuals with broad and potent neutralizing activity identified by using a high-throughput neutralization assay together with an analytical selection algorithm. J Viro/ 83, 7337-7348, doi:10.1128/JVI.00110-09 (2009).

39 Walker, L. M. \& Burton, D. R. Passive immunotherapy of viral infections: 'super-antibodies' enter the fray. Nat Rev Immuno/ 18, 297-308, doi:10.1038/nri.2017.148 (2018).

40 Hadfield, J. et al. Nextstrain: real-time tracking of pathogen evolution. Bioinformatics 34, 41214123, doi:10.1093/bioinformatics/bty407 (2018).

41 Murin, C. D., Wilson, I. A. \& Ward, A. B. Antibody responses to viral infections: a structural perspective across three different enveloped viruses. Nat Microbio/4, 734-747, doi:10.1038/s41564-0190392-y (2019). 
42 Garcia-Beltran, W. F. et al. COVID-19 neutralizing antibodies predict disease severity and survival. medRxiv, doi:10.1101/2020.10.15.20213512 (2020).

43 Roltgen, K. et al. Defining the features and duration of antibody responses to SARS-CoV-2 infection associated with disease severity and outcome. Sci Immuno/ 5, doi:10.1126/sciimmunol.abe0240 (2020).

44 Suthar, M. S. et al. Rapid Generation of Neutralizing Antibody Responses in COVID-19 Patients. Cell Rep Med 1, 100040, doi:10.1016/j.xcrm.2020.100040 (2020).

45 Luchsinger, L. L. et al. Serological Assays Estimate Highly Variable SARS-CoV-2 Neutralizing Antibody Activity in Recovered COVID-19 Patients. J Clin Microbio/ 58, doi:10.1128/JCM.02005-20 (2020).

46 Oran, D. P. \& Topol, E. J. Prevalence of Asymptomatic SARS-CoV-2 Infection : A Narrative Review. Ann Intern Med 173, 362-367, doi:10.7326/M20-3012 (2020).

47 Andreano, E. et al. SARS-CoV-2 escape in vitro from a highly neutralizing COVID-19 convalescent plasma. bioRxiv, doi:10.1101/2020.12.28.424451 (2020).

48 Tegally, H. et al. Emergence and rapid spread of a new severe acute respiratory syndrome-related coronavirus 2 (SARS-CoV-2) lineage with multiple spike mutations in South Africa. MedRxiv, doi:10.1101/2020.12.21.20248640 (2020).

49 Thomson, E. C. et al. The circulating SARS-CoV-2 spike variant N439K maintains fitness while evading antibody-mediated immunity. BioRxiv, doi:10.1101/2020.11.04.355842 (2020).

50 Jackson, L. A. et al. An mRNA Vaccine against SARS-CoV-2 - Preliminary Report. N Engl J Med 383, 1920-1931, doi:10.1056/NEJMoa2022483 (2020).

51 Sasaki, S. et al. Limited efficacy of inactivated influenza vaccine in elderly individuals is associated with decreased production of vaccine-specific antibodies. J Clin Invest 121, 3109-3119, doi:10.1172/JCl57834 (2011).

52 Rogers, T. F. et al. Isolation of potent SARS-CoV-2 neutralizing antibodies and protection from disease in a small animal model. Science 369, 956-963, doi:10.1126/science.abc7520 (2020).

53 Lv, Z. et al. Structural basis for neutralization of SARS-CoV-2 and SARS-CoV by a potent therapeutic antibody. Science 369, 1505-1509, doi:10.1126/science.abc5881 (2020).

54 Prevost, J. et al. Cross-Sectional Evaluation of Humoral Responses against SARS-CoV-2 Spike. Cell Rep Med 1, 100126, doi:10.1016/j.xcrm.2020.100126 (2020). 
55 Korber, B. et al. Tracking Changes in SARS-CoV-2 Spike: Evidence that D614G Increases Infectivity of the COVID-19 Virus. Cel/ 182, 812-827 e819, doi:10.1016/j.cell.2020.06.043 (2020).

56 Weissman, D. et al. D614G Spike Mutation Increases SARS CoV-2 Susceptibility to Neutralization. Cell Host Microbe, doi:10.1016/j.chom.2020.11.012 (2020).

57 Amanna, I. J., Carlson, N. E. \& Slifka, M. K. Duration of humoral immunity to common viral and vaccine antigens. N Engl J Med 357, 1903-1915, doi:10.1056/NEJMoa066092 (2007).

58 Edridge, A. W. D. et al. Seasonal coronavirus protective immunity is short-lasting. Nat Med 26, 1691-1693, doi:10.1038/s41591-020-1083-1 (2020).

59 Huang, A. T. et al. A systematic review of antibody mediated immunity to coronaviruses: kinetics, correlates of protection, and association with severity. Nat Commun 11, 4704, doi:10.1038/s41467-02018450-4 (2020).

60 Cao, W. C., Liu, W., Zhang, P. H., Zhang, F. \& Richardus, J. H. Disappearance of antibodies to SARSassociated coronavirus after recovery. N Engl J Med 357, 1162-1163, doi:10.1056/NEJMc070348 (2007).

61 Klingler, J. et al. Role of IgM and IgA Antibodies in the Neutralization of SARS-CoV-2. medRxiv, doi:10.1101/2020.08.18.20177303 (2020).

62 Krammer, F. SARS-CoV-2 vaccines in development. Nature 586, 516-527, doi:10.1038/s41586-0202798-3 (2020).

63 Crawford, K. H. D. et al. Protocol and Reagents for Pseudotyping Lentiviral Particles with SARSCoV-2 Spike Protein for Neutralization Assays. Viruses 12, doi:10.3390/v12050513 (2020).

64 Kärber, G. Beitrag zur kollektiven Behandlung pharmakologischer Reihenversuche. NaunynSchmiedebergs Archiv für Experimentelle Pathologie und Pharmakologie 162, 480-483, doi:10.1007/bf01863914 (1931).

65 Spearman, C. The Method of 'Right and Wrong Cases' ('Constant Stimuli') without Gauss's Formulae. British Journal of Psychology, 1904-1920 2, 227-242, doi:10.1111/j.2044-8295.1908.tb00176.x (1908).

66 Corman, V. M. et al. Detection of 2019 novel coronavirus (2019-nCoV) by real-time RT-PCR. Euro Surveill 25, doi:10.2807/1560-7917.ES.2020.25.3.2000045 (2020).

67 Scutari, M. Learning Bayesian Networks with thebnlearnRPackage. Journal of Statistical Software 35, doi:10.18637/jss.v035.i03 (2010).

68 Lüdecke, D. ggeffects: Tidy Data Frames of Marginal Effects from Regression Models. Journal of Open Source Software 3, doi:10.21105/joss.00772 (2018). 
a
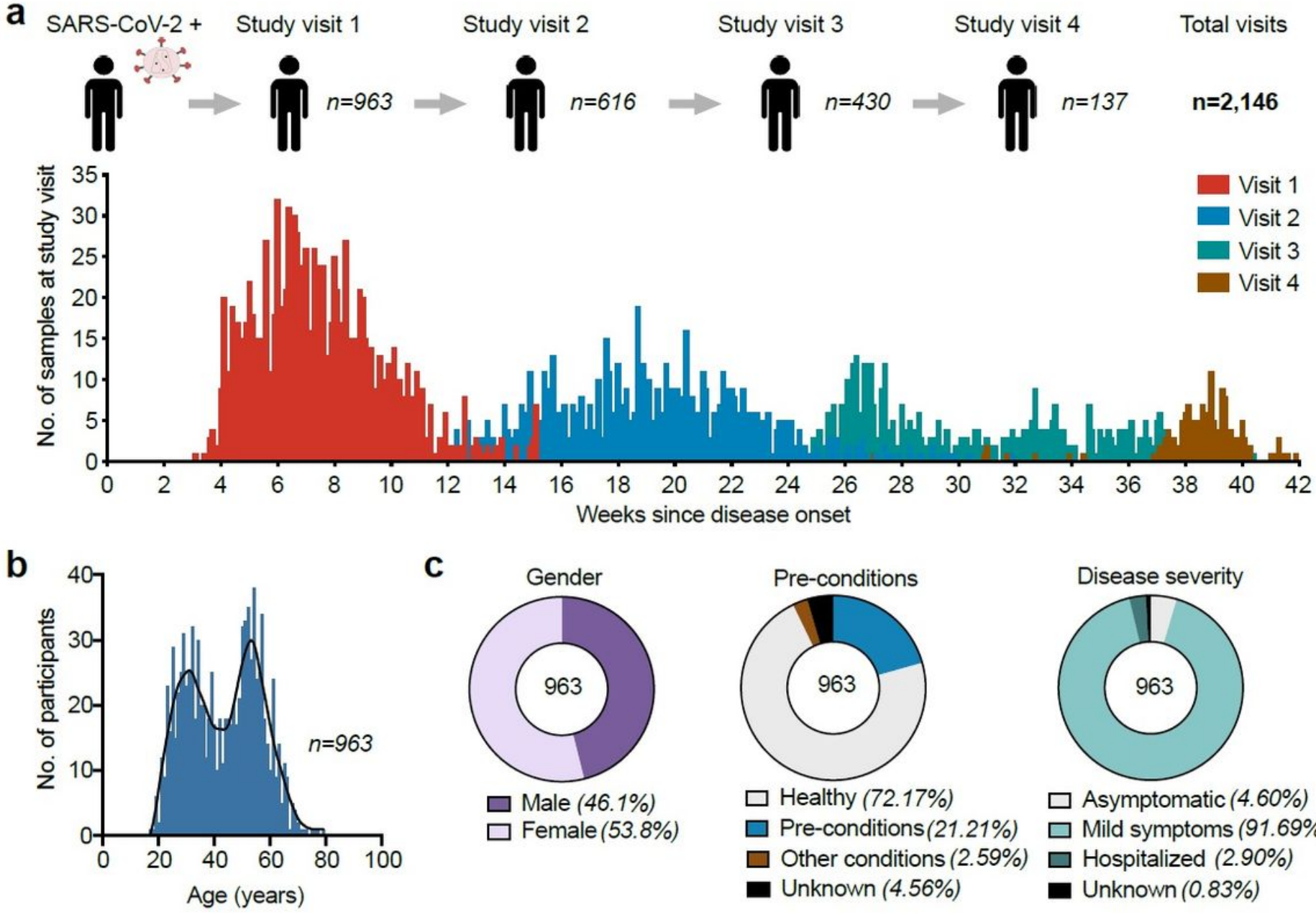

C
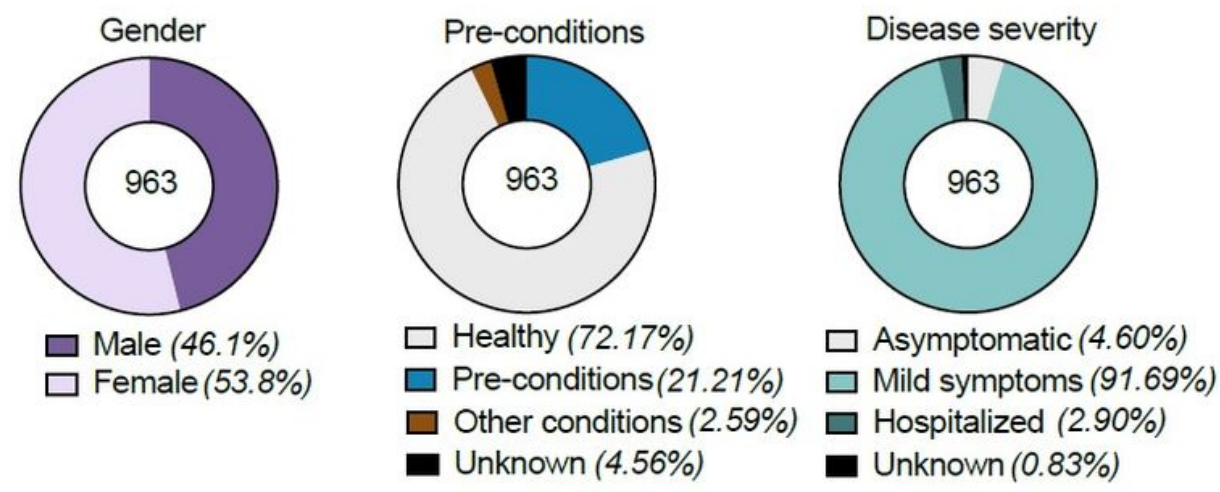

Figure 1

SARS-CoV-2 recovered cohort and study design a, Illustration depicting study timeline and number of individuals analyzed at each study visit. Graph represents sample collection time for participants in weeks since disease onset (symptom onset date or positive PCR date). b, age distribution of the cohort c, gender distribution, presence of pre-conditions and disease severity. 
a
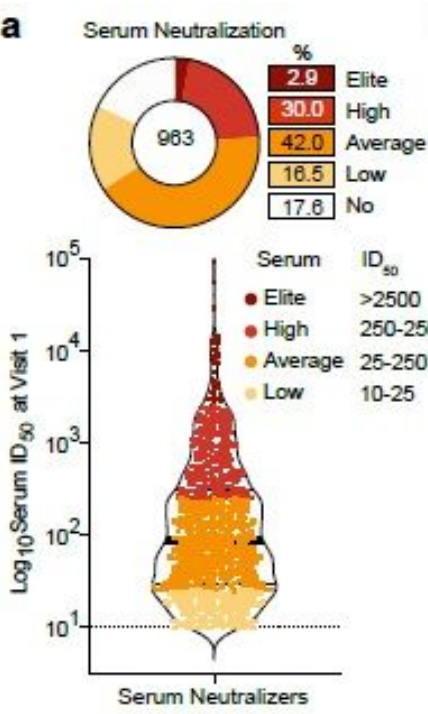

d

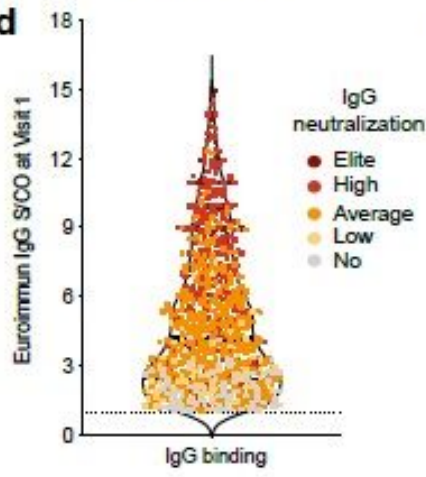

g

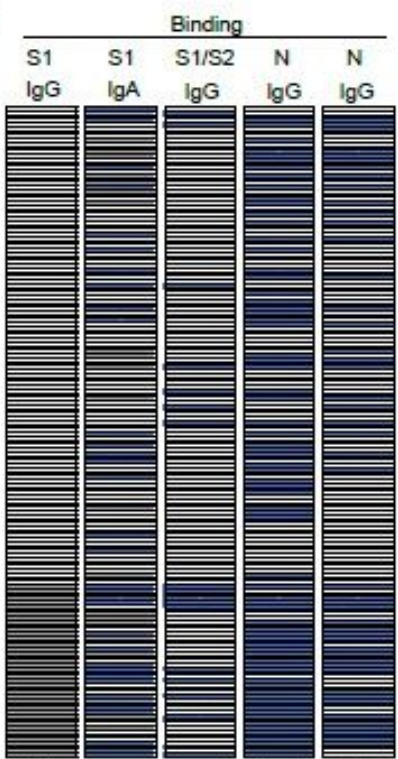

Euroimmun Diasorin Roche Abbott b

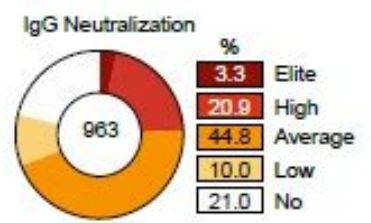

C IgG vs. Serum Neutralization

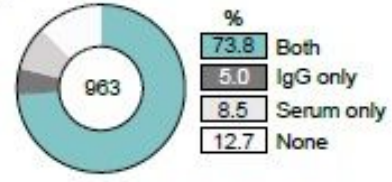

IgG vs. serum neutralization

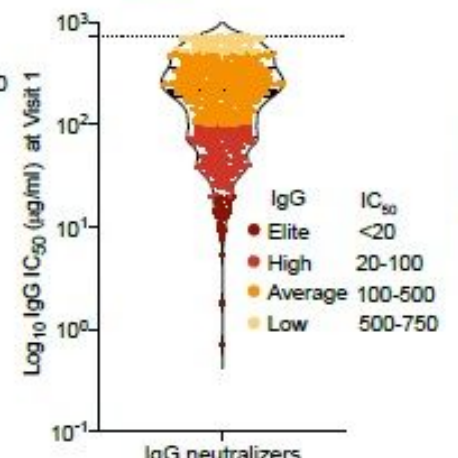

e

Euroimmun $\lg G$ ELISA

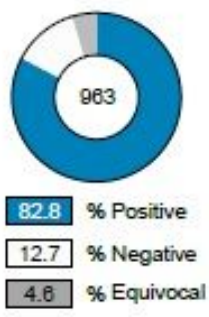

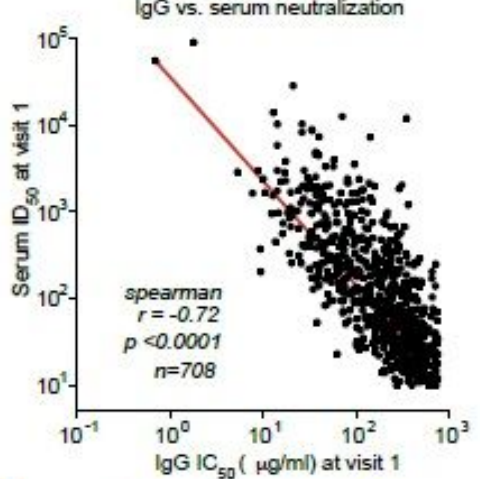

f

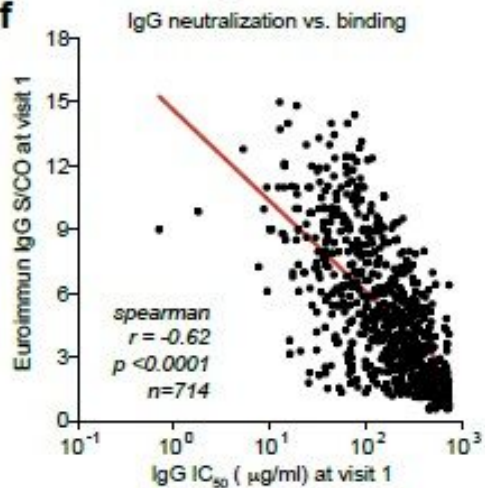

h
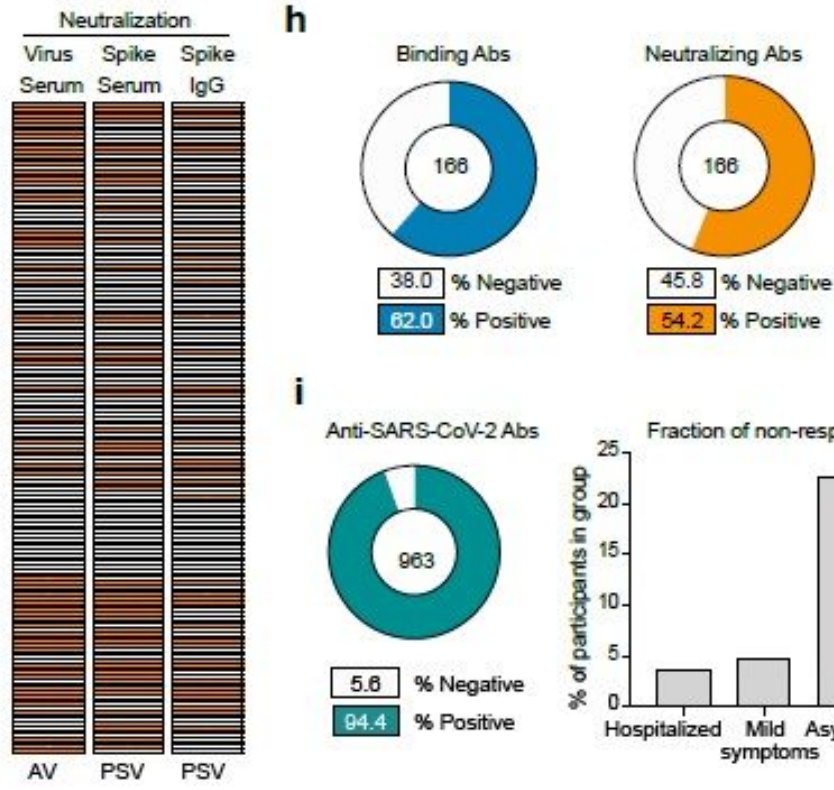

i
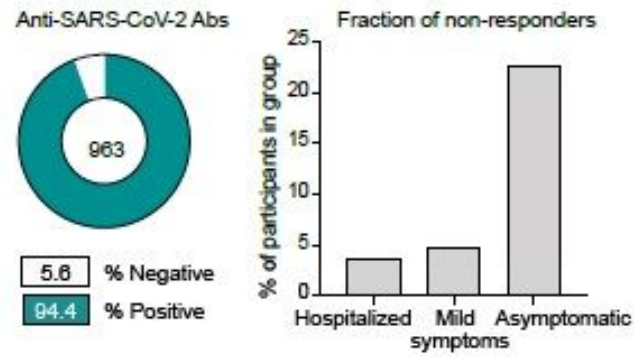

\section{Figure 2}

Neutralizing antibody response after recovery from SARS-CoV-2 infection a, pie chart illustrating fraction of serum neutralizers against Wu01 pseudovirus at study visit 1. Violin plot depicts serum ID50 values for the neutralizers ( $n=793)$, categorized based on serum ID50 titers. Dotted line represents the LOD (10-fold dilution) of the assay. b, pie chart depicting the fraction of IgG neutralization against Wu01 pseudovirus at study visit 1 . Violin plot depicts IgG IC50 values for the neutralizers $(n=760)$, categorized based on IgG 
IC50. Dotted line represents the LOD $(750 \mathrm{ug} / \mathrm{ml})$ of the assay. c, pie chart comparing fraction of samples with neutralization at serum and/or IgG level. Spearman correlation plot between serum ID50 and IgG IC50 values at study visit 1. d, violin plot of Euroimmun ELISA signal over cut-off (S/CO) ratios for antispike IgG. Dotted line represents LOD $(S / C O=1.1)$ of the assay. e, pie charts illustrating fraction of antispike IgG reactive individuals in the Euroimmun ELISA. $f$, spearman correlation between Euroimmun $\lg G$ S/CO and IgG IC50 at study visit 1. g, plot depicting binding against spike, Nucleocapsid (N) and neutralizing response against authentic virus (AV) and Wu01 pseudovirus (PSV) of the IgG negative fraction ( $n=166$ ) with each row representing 1 individual. h, pie charts showing total fraction of individuals with binding or neutralizing activity in the lgG-fraction from g. i, pie chart representing total combined binding and NAb response in the cohort $(n=963)$ and bar graph of the Ab-negative individuals based on disease severity. LOD, limit of detection 
a
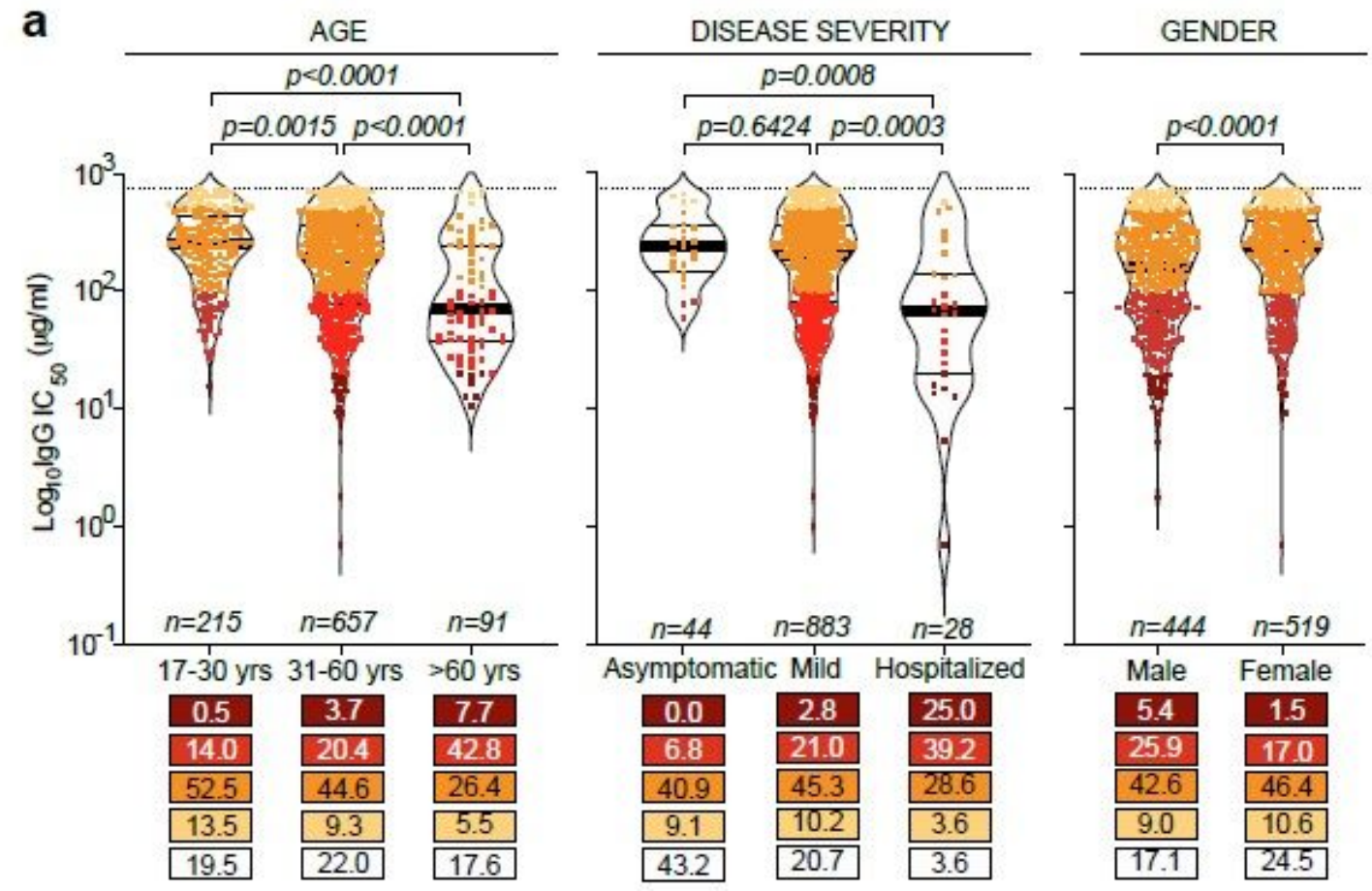

PRE-CONDITIONS

- Elite $<20 \mu \mathrm{g} / \mathrm{ml} \quad$ Average $100-500 \mu \mathrm{g} / \mathrm{ml}$

- High $20-100 \mu \mathrm{g} / \mathrm{ml}$ - Low $500-750 \mu \mathrm{g} / \mathrm{ml}$

\% Elite Neutralization $\square \%$ Average Neutralization

$\square \%$ High Neutralization $\square$ \% Low Neutralization

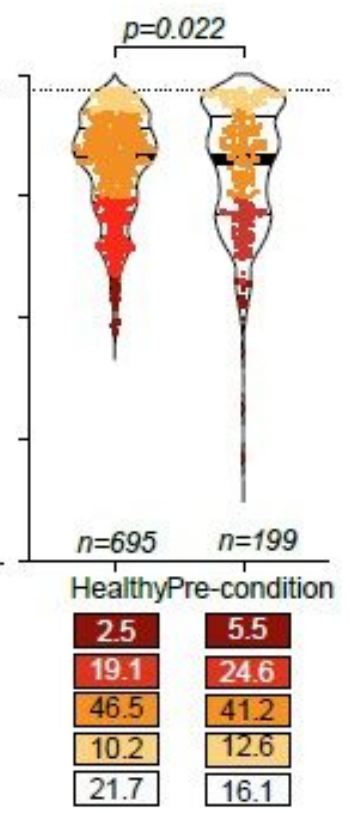

b

Feature selection for lgG neutralization model

C

SARS-CoV-2 IgG neutralization Bayesian network
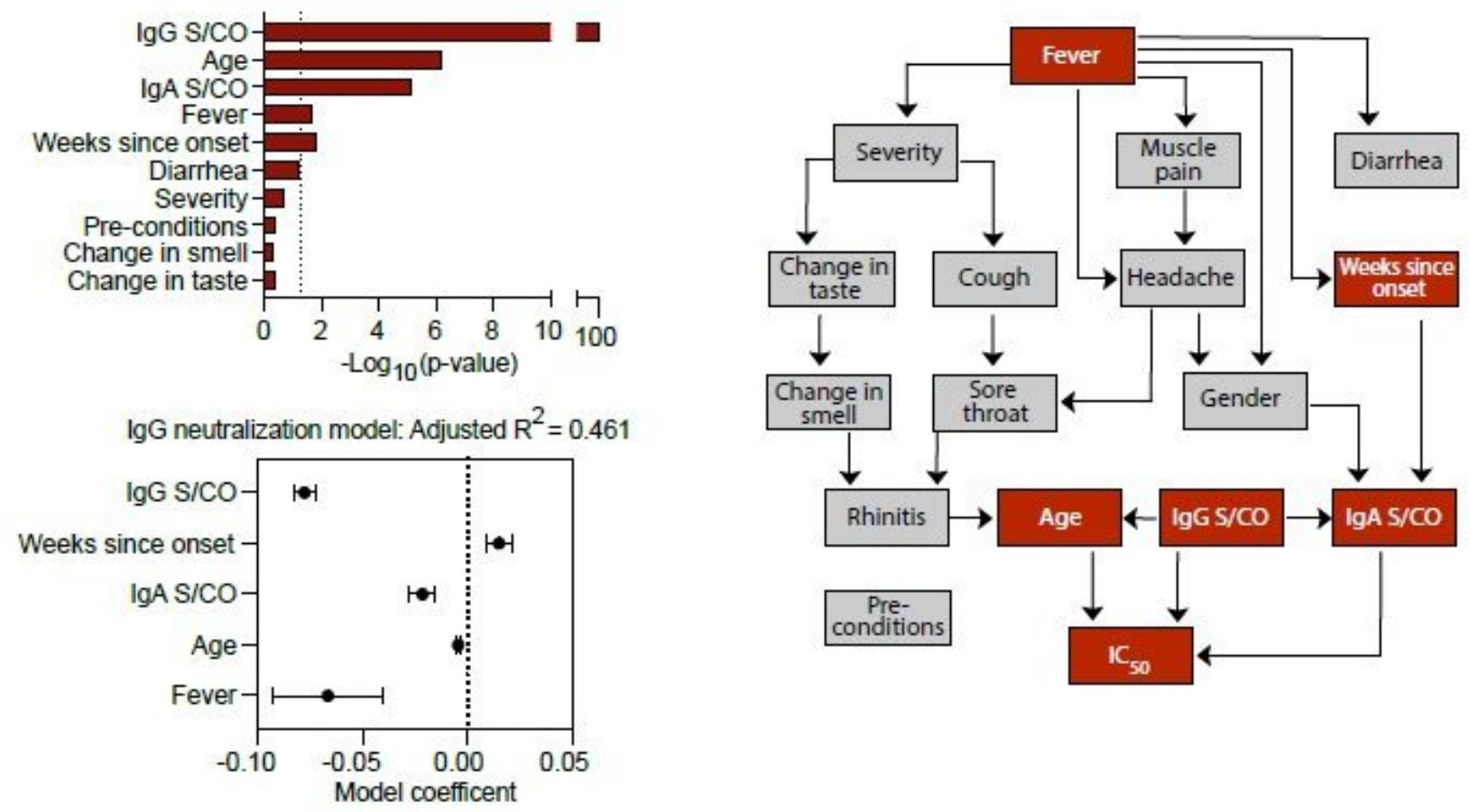

Figure 3

Correlates of neutralizing activity against SARS-CoV-2 a, violin plots depicting IgG neutralization IC50 values at study visit 1 against Wu01 pseudovirus, subdivided based on age, disease severity, gender and pre-conditions. Dotted line represents the limit of detection $(750 \mathrm{ug} / \mathrm{ml})$ of the assay. Statistical analysis was performed Kruskal-Wallis and Mann-Whitney tests. b, multiple linear regression model for predicting IgG IC50 using the features: Euroimmun S/CO, gender, age, disease severity, pre-conditions, weeks since 
infection and the 9 reported symptoms. Plot below depicts model coefficients to study the goodness of fit of the final IC50 prediction model. c, Bayesian network of the features predicting IgG IC50 are plotted using the bnlearn R package. The graph connects the features which are predictive of each other with IgG IC50 as sink.
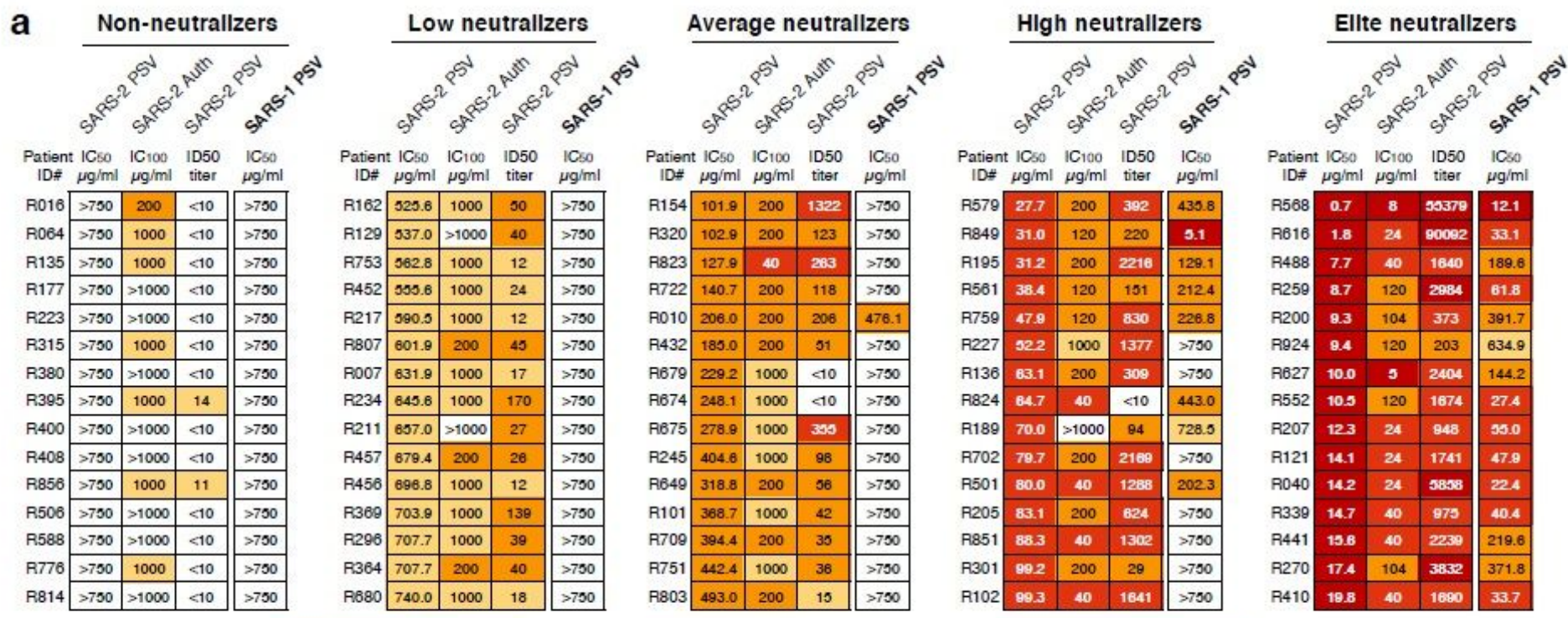

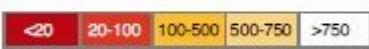
lgG IC $C_{s}(\mu \mathrm{g} / \mathrm{ml})$ against Pseudovirus (PSV)

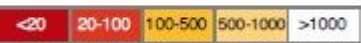
$\lg \mathrm{G} I \mathrm{C}_{1 \infty}(\mu \mathrm{g} / \mathrm{ml})$ against Authentic virus (Auth)

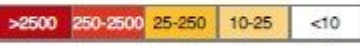

Serum ID so against Pseudovirus (PSV)

b

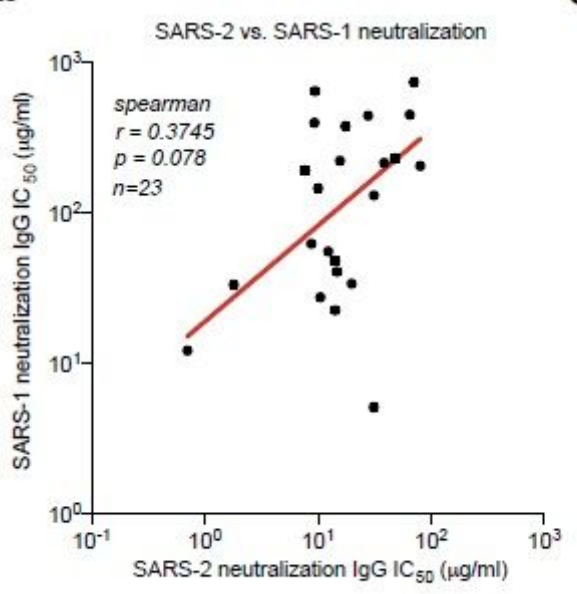

C

\begin{tabular}{|c|c|c|c|c|}
\hline $\begin{array}{l}\text { SARS-2-S } \\
\text { Viru\& name }\end{array}$ & $\begin{array}{l}\text { GISAID } \\
\text { identifier }\end{array}$ & $\begin{array}{l}\text { Region of origin } \\
\text { of atran }\end{array}$ & $\begin{array}{c}\text { A.A residue } \\
\text { mutation on } \\
\text { spike }\end{array}$ & $\begin{array}{c}\text { Location of } \\
\text { mutation on } \\
\text { spike }\end{array}$ \\
\hline Wu01 & EPLSL_40671 & Wuhan, China & - & - \\
\hline BowP1 & EPIJSL_406862 & Bevaria, Germany & D614G & S1 \\
\hline ARA36 & EPIJSL_418432 & Lyon, France & De38Y & S2; Heptad Repeat \\
\hline DRC94 & EPI」SL_417947 & Kinshasa, Africa & $\begin{array}{l}\text { D614G } \\
\text { L821I }\end{array}$ & $\begin{array}{l}\text { S1 and S2 (fusion } \\
\text { peptide) }\end{array}$ \\
\hline CA5 & EPIJSL_408010 & California, USA & $\mathrm{H} 49 \mathrm{Y}$ & $\begin{array}{c}\text { S1: N Terminal } \\
\text { domain }\end{array}$ \\
\hline NRW8 & EPIJSL_414508 & $\begin{array}{l}\text { Heinsberg. } \\
\text { Germany }\end{array}$ & S254F & $\begin{array}{l}\text { S1; N Terminal } \\
\text { domain }\end{array}$ \\
\hline
\end{tabular}

d

\section{Figure 4}

Cross-neutralization by SARS-CoV-2 elite neutralizers a, heat maps visualizing the neutralizing activity of 15 individuals from each neutralization category: Elite-, High-, Average-, Low-, and Non-neutralizers (total $\mathrm{n}=75$ ) against SARS-CoV-2-S pseudovirus, SARS-CoV-2 authentic virus and SARS-CoV (SARS-1) pseudovirus. b, Spearman correlation of IgG IC50 against SARS-2-S and SARS-1-S pseudovirus. c, details on the source and type of spike mutations in 6 global strains of SARS-CoV-2 generated and used in this study. $d$, heat map visualizing the IC50 values of 15 Elite-neutralizers against the 6 SARS-CoV-2 global spike variants from $c$. 
a

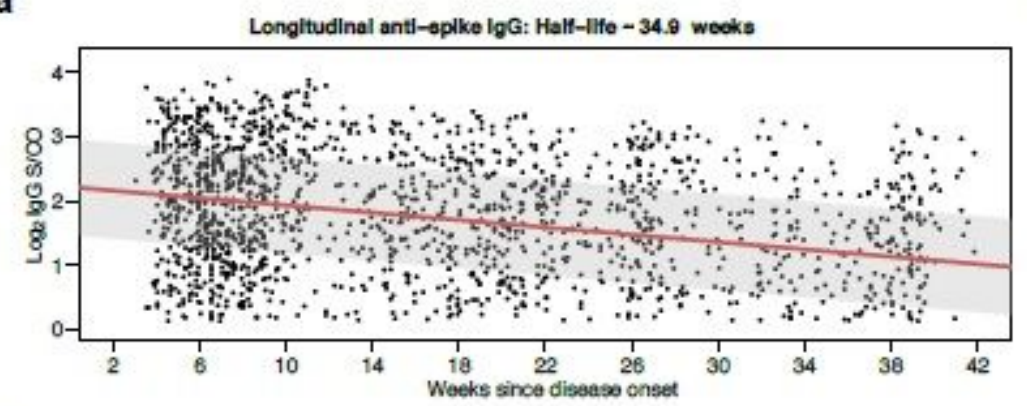

b

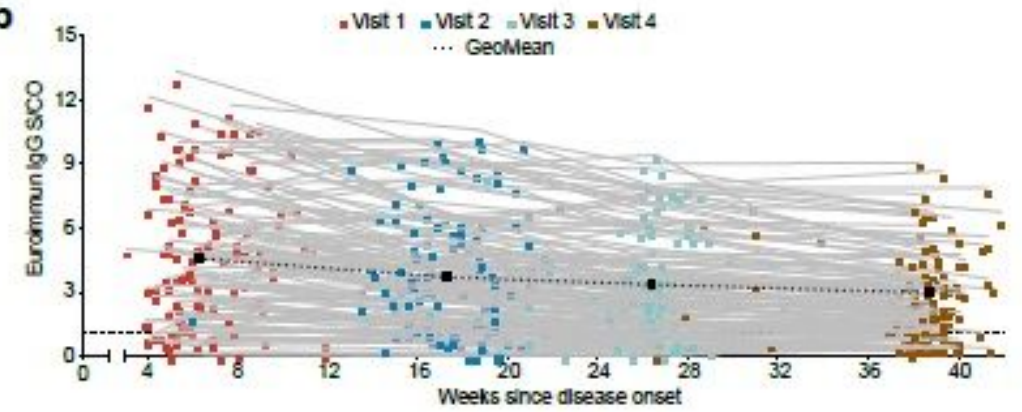

C

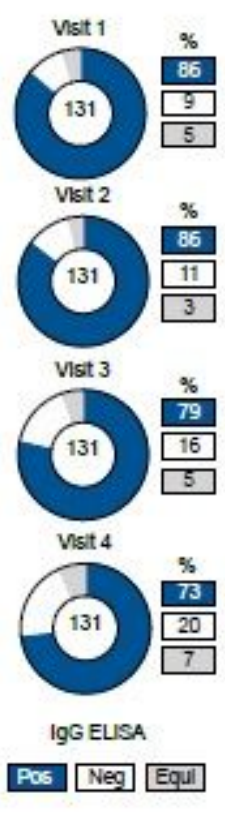

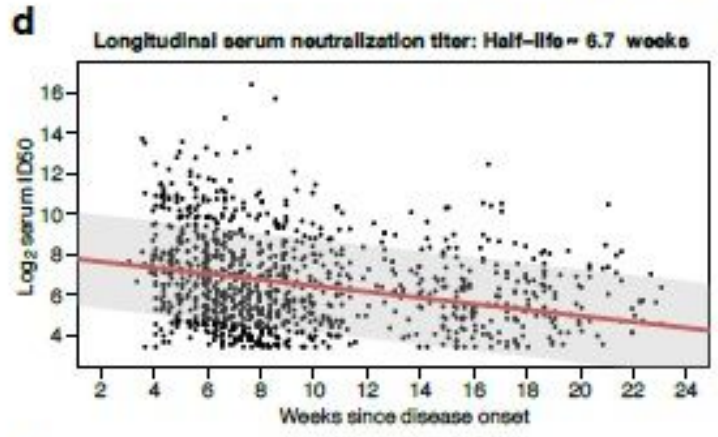

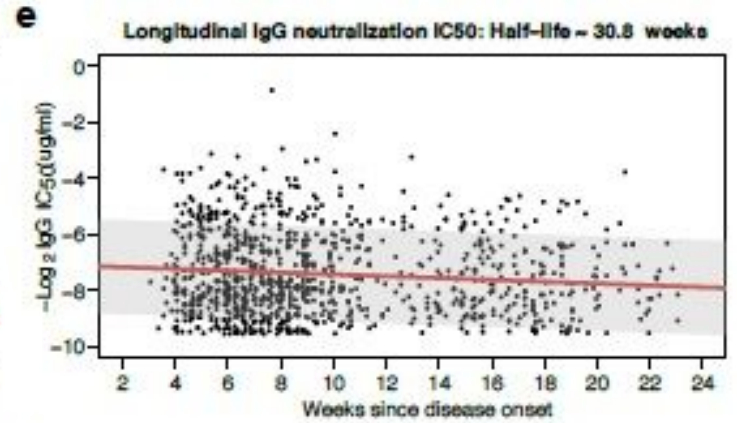

f
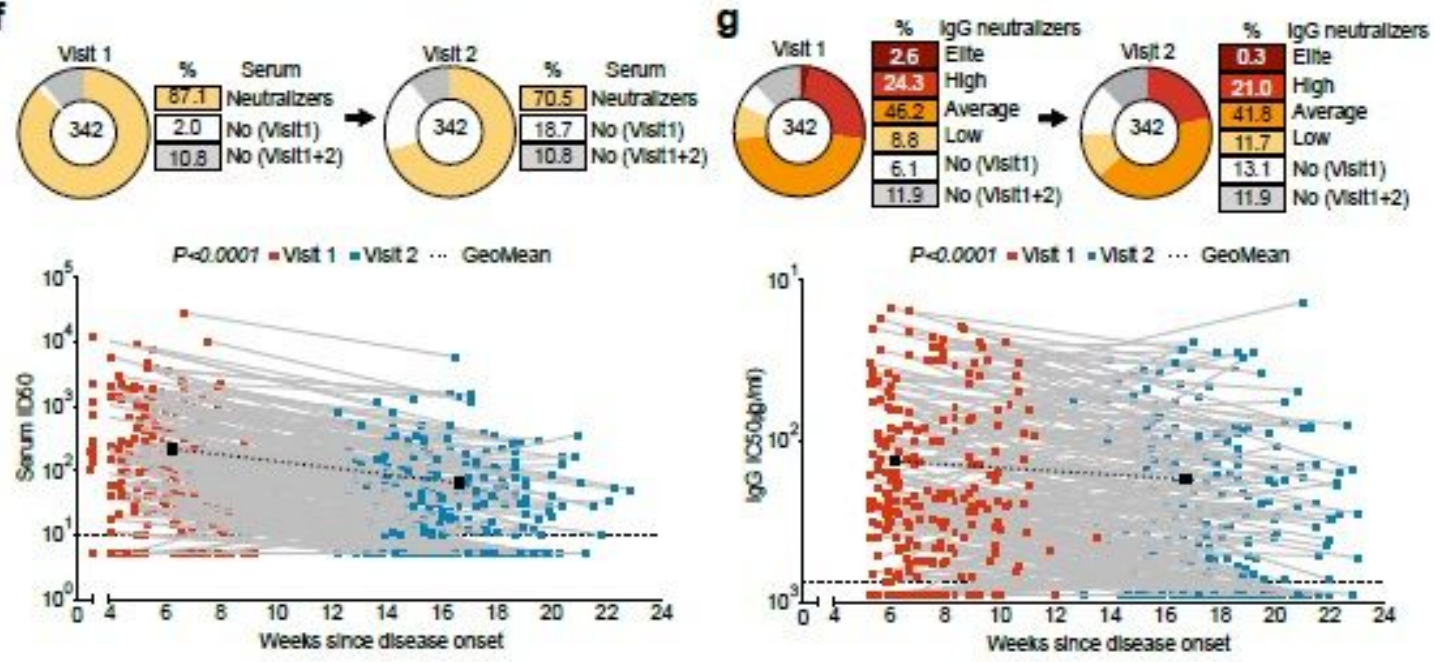

Figure 5

Longitudinal maintenance of anti-SARS-CoV-2 IgG antibody titers a, IgG ELISA ratios $(n=1,669)$ plotted against weeks since infection for half-life estimate of anti-spike lgG levels using a linear mixed-effects model. b, longitudinal mapping of IgG levels in 131 individuals from visit 1-4. Dot plots illustrate antibody titer against the weeks since infection to study visit 1 (red) and study visit 2 (blue). Geometric mean change shown in black. Dotted lines represent limit of detection (S/CO=1.1 for IgG ELISA). c, pie charts 
illustrate the change in the fraction of IgG ELISA positive (Pos), Negative (Neg) and Equivocal (Equi) samples $(n=131)$ between the study visits. $d$, serum ID50 values against Wu01 pseudovirus $(n=1,017)$ and e, IgG IC50 values against Wu01 pseudovirus $(n=996)$ plotted against weeks since infection for halflife estimate of the antibody levels using a linear mixed effects model. Longitudinal mapping of serum neutralization (f) and IgG neutralization (g) in 342 individuals at study visit 1 and 2. Serum and IgG nonneutralizers were assigned values of ID50 $=5$ and IC50 $=900$ for plotting. Dotted lines represent limit of detection (ID50 of 10 and IC50 of $750 \mathrm{ug} / \mathrm{ml}$ for serum and IgG neutralization assays). Pie charts illustrate the change in the fraction of serum neutralizers ( $\mathrm{f}$ ) and IgG neutralizers ( $\mathrm{g}$ ) in the samples $(n=342)$ between the study visits.

\section{Supplementary Files}

This is a list of supplementary files associated with this preprint. Click to download.

- VanshyllaetalExtendeddata.pdf

- flatNMEDA110981nreditorialpolicychecklistVanshyllaetal.pdf

- flatNMEDA110981nrreportingsummaryVanshyllaetal.pdf 